\title{
Fiberoptic, wire-guided king laryngeal tube exchange in trauma patients: a case series
}

\author{
Richard E. Galgon ${ }^{1 t^{*}}$, Aaron M. Joffe ${ }^{2 \dagger}$, Karl Willmann ${ }^{1 \dagger}$ and George A. Arndt ${ }^{1+}$ \\ ${ }^{*}$ Correspondence: galgon@wisc.edu \\ ${ }^{\dagger}$ These authors contributed equally to this work. \\ 'Department of Anesthesiology, University of Wisconsin School of Medicine and Public Health, Madison, WI, USA. \\ ${ }^{2}$ Department of Anesthesiology and Pain Medicine, University of Washington-Harborview Medical Center, Seattle, WA, USA.
}

\begin{abstract}
The laryngeal tube is increasingly being used for prehospital airway rescue. However, its exchange for an endotracheal tube can be challenging and risky. No best technique to achieve this end is yet accepted. We report three trauma cases where a fiberoptic, wire-guided, laryngeal tube-to-endotracheal tube exchange using the Arndt Exchange Catheter Set was performed. Three patients with multiple traumatic injuries presented to the emergency department with a laryngeal tube, with or without a gastric port, in place. All were in cervical stabilization collars. One had blood and gastric contents filling the ventilation tube and gastric port, respectively. One was undergoing cardiopulmonary resuscitation. In all cases, a fiberoptic bronchoscope and an Arndt Exchange Catheter Set were used to successfully exchange the laryngeal tube for an endotracheal tube without loss of airway control. In each case, the technique allowed continuous ventilation and oxygenation of the patient nearly throughout the entire exchange process. In conclusion, fiberoptic, wire-guided airway management using the Arndt Exchange Catheter Set can be successfully used to exchange the laryngeal tube for an endotracheal tube in trauma patients and provides a technique that maintains relative airway control throughout the entire exchange procedure, while providing nearly continuous ventilation and oxygenation.
\end{abstract}

Keywords: laryngeal tube, wire-guided, exchange

\section{Introduction}

The laryngeal tube (LT, King Systems, Noblesville, IN, USA), with and without a gastric port (LTS-D ${ }^{\mathrm{TM}}$ and LT-D ${ }^{\mathrm{TM}}$, respectively), is increasingly utilized for prehospital airway rescue [1-5]. Definitive care, however, often requires its exchange for an endotracheal tube (ETT), which may be challenging and risky [6]. No best technique, however, is yet known. Use of the Arndt Airway Exchange Catheter Set (AECS, Cook Medical Bloomington, IN, USA) for this purpose has been suggested [7], but no published reports currently exist. We report three trauma cases where prehospital endotracheal intubation (ETI) failure was rescued with an LT and a fiberoptic, wire-guided, LT-to-ETT exchange using the AECS was performed.

\section{Case series}

\section{Case 1}

A 68 year-old man, body mass index (BMI) $32.3 \mathrm{~kg} / \mathrm{m}^{2}$, sustained a parietal scalp laceration, frontal subdural hematoma, and multiple spine fractures in a motor vehicle accident. Emergency medical services (EMS) personnel attempted ETI four times without success at the scene for a Glasgow Coma Score (GCS) of 7; twice by direct laryngoscopy, once by videolaryngoscopy (Glidescope ${ }^{\circ}$, Verathon, Bothell, WA, USA), and once blindly through a S.A.L.T. airway (Microtek Medical, Columbus, MS, USA). Ultimately, a size 5
LT-D ${ }^{\mathrm{TM}}$ was successfully placed and the patient arrived in the emergency department (ED) unresponsive and supine on a backboard in a cervical stabilization collar.

\section{Case 2}

A 45 year-old man, BMI $42.9 \mathrm{~kg} / \mathrm{m}^{2}$, sustained frontal and parieto-temporal hemorrhagic cerebral contusions and multiple facial fractures in a motorcycle accident. EMS personnel and emergency physicians performed more than five ETI attempts using direct laryngoscopy at the scene and at an outside hospital (OSH). Prior to transport from the $\mathrm{OSH}$, an aeromedical team performed two additional unsuccessful attempts at ETI; once by videolaryngoscopy (Glidescope ${ }^{\circ}$ ) and once blindly through a S.A.L.T. airway. Ultimately, a size 4 LTS-D ${ }^{\text {TM }}$ was successfully placed. The patient arrived in the ED unresponsive and supine on a backboard with a cervical stabilization collar in place. Edema with ecchymosis distorted the right periorbital and mid-face anatomy. He was tachycardic, hypertensive, and hypoxic with an oxygen saturation in the 70s. Gastric contents and blood filled the gastric port and ventilation tube of the LTS- $\mathrm{D}^{\mathrm{TM}}$, respectively. Upon suctioning the ventilation tube, his oxygen saturation rose to $98 \%$.

\section{Case 3}

A 43 year-old woman, BMI $24.1 \mathrm{~kg} / \mathrm{m}^{2}$, sustained a closed-head 


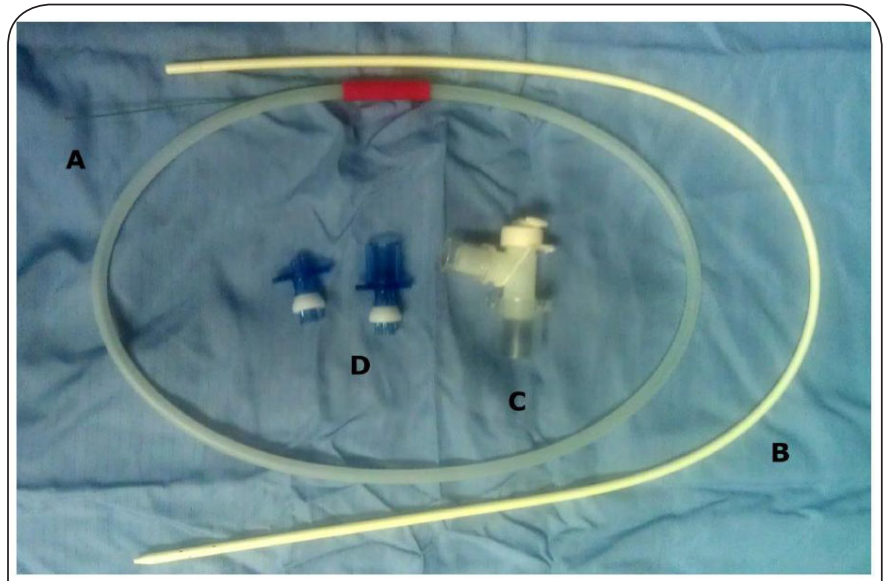

Figure 1. Components of the Arndt Exchange Catheter Set. (A) Flexible tip Amplatz wire. (B) Exchange catheter. (C) Bronchoscope airway adapter. (D) Rapi-fit ${ }^{\oplus}$ adapters.

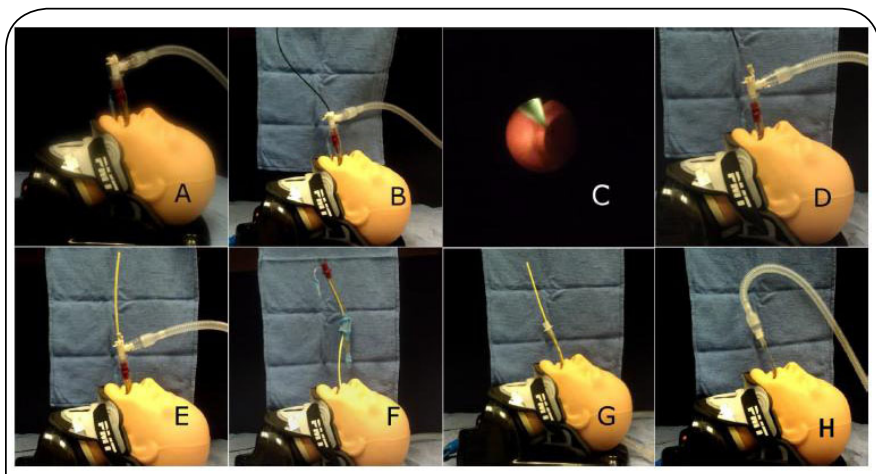

Figure 2. LT-to-ETT Exchange Technique: (A) Place the AECS airway adapter on the proximal end of the LT airway tube and ventilate the patient through the sidearm connector. (B) Guide a flexible FOB through the LT ventilation port and into the trachea. (C) Pass the AECS wire through the FOB working channel until visualized in the trachea. (D) Remove the FOB over the wire, while maintaining the position of the wire in the airway. (E) Place the AECS exchange catheter over the AECS wire. (F) Deflate the LT cuffs and remove it over the wire/ catheter unit. (G) Advance an ETT over the wire/catheter unit to an appropriate depth. (H) Remove the wire/catheter combination and verify correct ETT placement by bronchoscopic examination.

injury and femur fracture after being struck as a pedestrian by a moving vehicle. EMS records were unclear regarding her prehospital airway management. Nonetheless, she arrived in the ED in full cardiopulmonary arrest with a size 4 LT-D ${ }^{\mathrm{TM}}$ in situ, supine on a backboard in a cervical stabilization collar, undergoing cardiopulmonary resuscitation.

In each above case, the anesthesia service was called and asked to replace the LT with an ETT. This was performed using a fiberoptic bronchoscope (FOB) and the AECS (Figure 1). Before initiating the exchange, the gastric port (if present) and ventilation tube of the LT were suctioned with 18 Fr suction catheters. The AECS bronchoscope airway adapter was then placed on the LT, and mechanical ventilation and oxygenation were continued using the adapter sidearm (Figure 2, Panel A). With an assistant stabilizing the LT, either a Pentax Fl-13P (distal tip OD $4.2 \mathrm{~mm}$; working channel diameter $1.8 \mathrm{~mm}$ ) or FB-10V (distal tip OD $3.4 \mathrm{~mm}$; working channel diameter $1.2 \mathrm{~mm}$ ) flexible FOB (Pentax Medical, Montvale, NJ, USA) was advanced into the LT ventilation tube (Figure 2, Panel B) until the main ventilation port was identified and the glottic opening could be visualized through the port. Once the glottic opening could be visualized through the main LT ventilation port, the FOB was advanced through the glottic opening and into the trachea to the level of the carina. The AECS wire was then advanced through the FOB working channel until visualized in the lower trachea or main stem bronchus (Figure 2, Panel C). The FOB was then removed over the wire, while maintaining the wire in the same relative position (Figure 2, Panel D). The AECS exchange catheter was then advanced through the LT over the wire (Figure 2, Panel E), and the LT was removed over the catheter/ wire combination (Figure 2, Panel F). An ETT was then advanced over the catheter/wire combination (Figure 2, Panel G), and the catheter/wire combination was removed. Correct ETT placement was confirmed by visualizing the ETT tip in the trachea above the carina. Secondarily, bilateral breath sounds, and a positive end-tidal carbon dioxide tracing were confirmed. In each case, the patients' oxygen saturations remained greater than $95 \%$ throughout the exchange procedure.

\section{Discussion}

Out-of-hospital airway management requires ETI in approximately $0.5 \%$ of EMS activations and $12-15 \%$ of trauma patients [8-10]. Prehospital ETI success rates range from $48 \%$ to $98 \%$ and depend on patient characteristics, setting, pharmacologic adjunct use, and EMS skill [8-12]. Failed ETI is rescued using alternative airway devices, a return to bag-mask ventilation, or cricothyrotomy [10-11]. Due to its ease of insertion, short insertion time, and ventilatory effectiveness, the LT is becoming preferred by EMS personnel $[1,3]$. Its exchange for an ETT, however, may be challenging and risky [6].

We describe the first applications of fiberoptic, wireguided airway management using the AECS for LT-to-ETT exchange in three trauma patients. Previously, the use of this technique has been documented for LMA-Classic ${ }^{\mathrm{TM}}$ and LMA-Proseal $^{\mathrm{TM}}$ (LMA North America, San Diego, CA, USA) exchange in the operating room and intensive care unit [13-15]. However, its successful application for exchanging an LT has not been previously reported. We believe the application of this technique for managing an LT-to-ETT exchange makes an important contribution to the airway manager's armamentarium for management of the LT placed in the prehospital setting for airway rescue in trauma patients.

The AECS is a ready-made kit containing (1) a $140 \mathrm{~cm}, 0.35$ $\mathrm{mm}$ diameter, flexible tip, polytetrafluoroethylene coated Amplatz wire, (2) a $70 \mathrm{~cm}, 14 \mathrm{Fr}$ (4.7 mm OD) radiopaque, exchange catheter with distance markings every centimeter to $35 \mathrm{~cm}$, (3) a bronchoscope airway adapter, and (4) Rapi- 
fit $^{\circ}$ adapters that allow oxygenation through the exchange catheter if necessary. The wire is thin enough to pass through all, but neonatal fiberoptic bronchoscopes, and the exchange catheter accommodates ETTs with internal diameters as small as $5 \mathrm{~mm}$.

Besides the fiberoptic, wire-guided technique we describe, Genzwuerker et al., reported successful first-attempt, exchange of an earlier generation of the LT in eight of ten patients under general anesthesia in the operating room using an Aintree Intubation Catheter (AIC, Cook Deutschland, Moenchengladbach, Germany) mounted on a flexible fiberoptic scope [16]. One failure occurred when the glottic opening could not be visualized from the termination of the LT airway tube despite attempts at manipulating the device. The other failure occurred due to dislodgement of the AIC from the trachea during the exchange procedure with consequent esophageal intubation. Lutes et al., attempted LT exchange in a manikin and a cadaver by blindly passing a gum elastic bougie through the $L T$, but this technique was unsuccessful [17]. In the manikin, failure occurred due to bougie passage into the esophagus and hang-up on the lateral airway structures, while the right aryepiglottic fold was perforated in the cadaver. Finally, Khaja et al., described six patients in whom an LT had been placed for airway rescue [6]. Attempts at LT-to-ETT exchange using direct laryngoscopy and/or flexible fiberoptic bronchoscopy adjacent to the oropharyngeal balloon failed in five patients due to soft tissue and/or tongue swelling, which limited laryngeal structure visualization despite partial or full deflation of the oropharyngeal balloon. These five patients required emergent tracheostomy for definitive airway management.

Application of wire-guided airway management using the AECS for LT-to-ETT exchange allows unhurried, fiberoptic instrumentation of the airway with continuous oxygenation and ventilation through the sideport of the AECS bronchoscope airway adapter up to the point of $L T$ removal. Compared with fiberoptic-guided AIC exchange, the handling characteristics of the fiberoptic bronchoscope are improved as its movement is not constrained or stiffened by a coaxially mounted exchange catheter, and bronchoscopes with outer diameters up to 6.27 $\mathrm{mm}$ can be used. The AECS exchange catheter provides a longer working length compared to the AIC $(70 \mathrm{~cm}$ vs. 56 $\mathrm{cm}$ ) and relative airway control is maintained with the AECS wire even if the AECS exchange catheter is inadvertently withdrawn. Further, ETTs with internal diameters as small as $5 \mathrm{~mm}$ ( $\mathrm{vs}$. $\geq 7 \mathrm{~mm}$ ) can be placed over the AECS exchange catheter, while even smaller ETTs can be placed by omitting the AECS exchange catheter and railroading the ETT directly over the AECS wire in a manner similar to wire-guided ETT placement described by Hasan and Black [18]. If time permits, the FOB, ETT, and AECS components should be lubricated with a medical grade, silicone oil spray or another alternative lubricant to facilitate component sliding during the exchange procedure.
As with all fiberoptic-based techniques, this technique may be limited in heavily soiled or hemorrhaging airway conditions where irrigation and/or suction cannot provide adequate visualization of the airway structures. Further, it requires the operator to be knowledgeable and skilled in fiberoptic bronchoscopy. The reader should note that the exchanges we describe were all performed by either seniorlevel anesthesia residents or an attending anesthesiologist; all of whom routinely use flexible bronchoscopy and the AECS for difficult airway management in their practices. Although we were unable to assess from the medical records we reviewed the explicit time required for exchange, we have found it takes attending anesthesiologists an average (SD) of 112 (28) seconds to perform the exchange procedure in a manikin with a mean (SD) apnea time during the procedure of 38 (13) seconds (unpublished data).

In summary, the application of fiberoptic, wire-guided airway management using the AECS for the successful LTto-ETT exchange is presented. Given the ability to maintain relative airway control and to oxygenate and ventilate the patient nearly throughout the entire exchange procedure compared to available alternative techniques, we recommend use of this technique when presented with an in situ LT requiring exchange to an ETT.

\section{Competing interests}

REG, AMJ and KW have no competing interests. George A. Arndt, MD., is a paid consultant to Cook Medical and receives royalties for the Arndt Exchange Catheter Set .

\section{Authors' contributions}

REG: Involved in the case(s). Reviewed the charts. Prepared, revised, and approved the manuscript. AMJ: Involved in the case(s). Revised and approved the manuscript. KW: Involved in the case(s). Approved the manuscript. GAA: Involved in the case(s). Approved the manuscript.

\section{Publication history}

Received: 19-Nov-20102 Revised: 19-Dec-2012

Accepted: 23-Dec-2012 Published: 28-Dec-2012

\section{References}

1. Tumpach EA, Lutes $M$, Ford D and Lerner EB: The King LT versus the Combitube: flight crew performance and preference. Prehosp Emerg Care 2009, 13:324-8. | Article | PuMed

2. Russi CS, Hartley MJ and Buresh CT: A pilot study of the King LT supralaryngeal airway use in a rural lowa EMS system. Int J Emerg Med 2008, 1:135-8. | Article | PuMed Abstract | PubMed Full Text

3. Guyette FX, Wang $\mathrm{H}$ and Cole JS: King airway use by air medical providers. Prehosp Emerg Care 2007, 11:473-6. I Article I PuMed

4. Schalk R, Byhahn C, Fausel F, Egner A, Oberndorfer D, Walcher F and Latasch L: Out-of-hospital airway management by paramedics and emergency physicians using laryngeal tubes. Resuscitation 2010, 81:323-6. | Article | PuMed

5. Wiese $\mathrm{CH}$, Semmel T, Muller JU, Bahr J, Ocker $\mathrm{H}$ and Graf BM: The use of the laryngeal tube disposable (LT-D) by paramedics during out-ofhospital resuscitation-an observational study concerning ERC guidelines 2005. Resuscitation 2009, 80:194-8. | Article | PuMed

6. Khaja SF, Provenzano MJ and Chang KE: Use of the King LT for emergency 
Galgon et al. Journal of Anesthesiology and Clinical Science 2012, http://www.hoajonline.com/journals/pdf/2049-9752-1-15.pdf

airway management. Arch Otolaryngol Head Neck Surg 2010, 136:979-

82. | Article | PuMed

7. Matioc AA and Genzwuerker HV: Why go blind when you can see? J Emerg Med 2012, 42:702-3. | Article | PuMed

8. Wang HE, Balasubramani GK, Cook LJ, Yealy DM and Lave JR: Out-ofhospital airway management in the United States. Prehosp Emerg Care 2011, 15:338-46. | Article | PuMed

9. Bulger EM, Nathens AB, Rivara FP, MacKenzie E, Sabath DR and Jurkovich GJ: National variability in out-of-hospital treatment after traumatic injury. Ann Emerg Med 2007, 49:293-301. I Article I PuMed

10. Cobas MA, De la Pena MA, Manning R, Candiotti K and Varon AJ: Prehospital intubations and mortality: a level 1 trauma center perspective. Anesth Analg 2009, 109:489-93. | Article | PuMed

11. Hubble MW, Brown L, Wilfong DA, Hertelendy A, Benner RW and Richards ME: A meta-analysis of prehospital airway control techniques part I: orotracheal and nasotracheal intubation success rates. Prehosp Emerg Care 2010, 14:377-401. | Article | PuMed

12. Garza AG, Gratton MC, Coontz D, Noble E and Ma OJ: Effect of paramedic experience on orotracheal intubation success rates. J Emerg Med 2003, 25:251-6. | Article | PuMed

13. Arndt GA, Topp J, Hannah J, McDowell TS and Lesko A: Intubation via the LMA using a Cook retrograde intubation kit. Can J Anaesth 1998, 45:25760. | Article | PuMed

14. Matioc $A$ and Arndt GA: Intubation using the ProSeal laryngeal mask airway and a Cook airway exchange catheter set. Can J Anaesth 2001, 48:932. | Article | PuMed

15. Joffe $A M$, Arndt $G$ and Willmann K: Wire-guided catheter exchange after failed direct laryngoscopy in critically ill adults. J Clin Anesth 2010, 22:936. | Article | PuMed

16. Genzwuerker HV, Vollmer T and Ellinger K: Fibreoptic tracheal intubation after placement of the laryngeal tube. Br J Anaesth 2002, 89:733-8. | Article I PuMed

17. Lutes $M$ and Worman DJ: An unanticipated complication of a novel approach to airway management. J Emerg Med 2010, 38:222-4. | Article I PuMed

18. Hasan MA, Black $A E$ : A new technique for fiberoptic intubation in children. Anaesthesia. 1994, 49:1031-3. | Article 